\title{
TRIGONOMETRIC APPROXIMATION IN GENERALIZED LEBESGUE SPACES $L^{p(x)}$
}

\author{
Ali GUVEN AND DANIYAL M. ISRAFILOV
}

Abstract. The approximation properties of Nörlund $\left(N_{n}\right)$ and Riesz $\left(R_{n}\right)$ means of trigonometric Fourier series are investigated in generalized Lebesgue spaces $L^{p(x)}$. The deviations $\left\|f-N_{n}(f)\right\|_{p(x)}$ and $\left\|f-R_{n}(f)\right\|_{p(x)}$ are estimated by $n^{-\alpha}$ for $f \in \operatorname{Lip}(\alpha, p(x))(0<\alpha \leqslant 1)$.

Mathematics subject classification (2010): 41A25, 42A10, 46E30.

Keywords and phrases: Generalized Lebesgue space, Lipschitz class, modulus of continuity, Nörlund mean, Riesz mean.

\section{REFERENCES}

[1] P. ChandRA, Approximation by Nörlund operators, Mat. Vestnik 38 (1986), 263-269.

[2] P. Chandra, Functions of classes $L_{p}$ and Lip $(\alpha, p)$ and their Riesz means, Riv. Mat. Univ. Parma (4) 12 (1986), 275-282.

[3] P. Chandra, A note on degree of approximation by Nörlund and Riesz operators, Mat. Vestnik 42 (1990), 9-10.

[4] P. ChandRA, Trigonometric approximation of functions in $L_{p}$-norm, J. Math. Anal. Appl. 275 (2002), $13-26$.

[5] D. Cruz-Uribe, A. Fiorenza, C. J. Neugebauer, The maximal function on variable $L^{p}$ spaces, Ann. Acad. Sci. Fenn. Math. 28 (2003), 223-238, and 29 (2004), 247-249.

[6] R. A. Devore, G. G. Lorentz, Constructive Approximation, Springer-Verlag (1993).

[7] L. Diening, M. RuZickA, Calderon-Zygmund operators on generalized Lebesgue spaces $L^{p(x)}$ and problems related to fluid dynamics, J. Reine Angew. Math. 563 (2003), 197-220.

[8] L. Diening, Maximal function on generalized Lebesgue spaces $L^{p(x)}$, Math. Inequal. Appl. 7 (2004), 245-253.

[9] D. E. Edmunds, J. Lang, A. Nekvinda, On $L^{p(x)}$ norms, Proc. R. Soc. Lond. A 455 (1999), $219-225$.

[10] X. FAN, D. ZhaO, On the spaces $L^{p(x)}(\Omega)$ and $W^{m, p(x)}(\Omega)$, J. Math. Anal. Appl. $263(2001), 424$ 446.

[11] A. Guven, Trigonometric approximation of functions in weighted $L^{p}$ spaces, Sarajevo J. Math 5 (17) (2009), 99-108.

[12] D. M. IsRafilov, V. KokilashVILI, S. SAMKo, Approximation in weighted Lebesgue and smirnov Spaces with variable exponents, Proc. A. Razmadze Math. Inst. 143 (2007), 25-35.

[13] O. KovaciK, J. RakosniK, On spaces $L^{p(x)}$ and $W^{k, p(x)}$, Czechoslovak Math. J. 41 (1991), 592 618.

[14] N. X. Ky, Moduli of Mean Smoothness and Approximation with $A_{p}$-weights, Annales Univ. Sci. Budapest 40 (1997), 37-48.

[15] L. LeINDLER, Trigonometric approximation in $L_{p}$-norm, J. Math. Anal. Appl. 302 (2005), 129-136.

[16] R. N. Mohapatra, D. C. RusSELl, Some direct and inverse theorems in approximation of functions, J. Austral. Math. Soc. (Ser. A) 34 (1983), 143-154.

[17] A. NeKVIndA, Hardy-Littlewood maximal operator on $L^{p(x)}(\mathbb{R})$, Math. Inequal. Appl. 7 (2004), 255-265.

[18] L. PICK, M. RUZICKA, An example of a space $L^{p(x)}$ on which the Hardy-Littlewood maximal operator is not bounded, Expo. Math. 19 (2001), 369-371. 
[19] E. S. QUADE, Trigonometric approximation in the mean, Duke Math. J. 3 (1937), 529-542.

[20] I. I. SHARAPUDINOV, Uniform boundedness in $L^{p}(p=p(x))$ of some families of convolution operators, Math. Notes 59 (1996), 205-212.

[21] I. I. SHARAPUDINOV, Some problems in approximation theory in the spaces $L^{p(x)}$, (Russian), Analysis Mathematica 33 (2007), 135-153.

[22] A. Zygmund, Trigonometric Series, Vol I, Cambridge Univ. Press, 2nd edition, (1959). 